\title{
Visualizing Solid-State Molecular Motion by a Common Structural Determination Technique
}

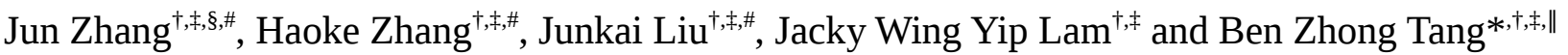 \\ ${ }^{\dagger}$ Department of Chemistry, Hong Kong Branch of Chinese National Engineering Research Center for \\ Tissue Restoration and Reconstruction and Institute for Advanced Study, The Hong Kong University \\ of Science and Technology, Clear Water Bay, Kowloon, Hong Kong SAR, China \\ ${ }^{\ddagger}$ HKUST-Shenzhen Research Institute, No. 9 Yuexing 1st Rd, South Area, Hi-tech Park, Nanshan, \\ Shenzhen 518057, China \\ §School of Materials and Chemical Engineering, Anhui Jianzhu University, Hefei 230601, China \\ "Center for Aggregation-Induced Emission, State Key Laboratory of Luminescent Materials and \\ Devices, SCUT-HKUST Joint Research Institute, South China University of Technology, Tianhe Qu, \\ Guangzhou 510640, China
}

\section{Supporting Information}

\begin{abstract}
Solid-state molecular motion plays a vital role in many advanced technologies. However, visualization of these processes is still challenging due to the limitation of characterization method. In this work, a common structural determination technique, single-crystal X-ray diffraction, is applied to "see" the static and dynamic molecular motions in tetraphenylethylene (TPE) which exhibits aggregation-induce emission (AIE) effect. Five kinds of motions, stretching, torsion, twisting, rocking, and wagging are observed and analyzed. As the static molecular motions, the middle double bond in TPE becomes short and twisted and the peripheral phenyl rings as a whole tend to be more twisted with the raising of testing temperature (150 to $298 \mathrm{~K}$ ). Meanwhile, dynamic motions of phenyl-rings rocking and wagging are found to be more and more vigorous along with the increase of temperature. This work provides a platform for visualizing solid-state molecular motion based on a common technique. It also affords direct evidence for the AIE mechanism of restriction of intramolecular motions.
\end{abstract}

Motions play essential roles in many different areas, e.g., chemistry, biology, and engineering. ${ }^{1,2}$ In the long process of human history, people have hardly ever stop exploring the laws of motion. After long-term and persistent efforts, physicists built lots of classical and quantum mechanics to describe the motions in various scales ranging from the macroscopic universe to microscopic quark, and also in different dimensions (e.g. 1D,
2D, and 3D). In essence, motions in molecular level determine the properties of materials. For example, the activity of molecular motion has a direct relationship with the existing forms of materials. Generally, gaseous molecule shows a vigorous motion and the molecular motion is fairly restricted in the solid state. Undoubtedly, molecular motions, especially in the solid state, are capable of doing lots of useful works. ${ }^{3-7}$ However, it is still a tough task to visualize and manipulate the solid-state molecular motion which impedes its further applications.

Since the molecular motion is so important, researchers have investigated it for a long time and developed many methods to visualize this process, i.e. spectroscopies (nuclear magnetic resonance (NMR), photoluminescence (PL), and absorption etc.), $\frac{8-10}{10}$ and microscopies (atomic force microscopy (AFM), scanning tunneling microscope (STM), and cryogenic electron microscopy (cryo-

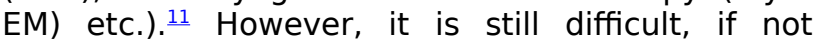
impossible, to realize the in-situ and real-time monitoring molecular motion in the solid state. For example, the obtained NMR spectra can only be utilized to speculate the molecular structure in one specific state. Although the STM can really "see" the molecular motion, it is only applicable to a single molecule in most cases which is more likely in gas phase. Moreover, these microscopic measurements are always expensive and complicated, and the sample needs to be pretreated and fixed before the characterization which may destroy their original structures. So, it is necessary and urgent to find a common structural 
determination method which can offer a platform to visualize the solid-state molecular motion.

Single-crystal X-ray diffraction (XRD) is a common and powerful technique to determine the molecular structure in atomic level. $\underline{\underline{12}}$ In general, the identified atoms position in XRD measurement are the average results represented as thermal ellipsoids. The location distribution in thermal ellipsoids is caused by the active molecular motion which is a thorny problem for researchers. In order to obtain an accurate structure, technicians will decrease the testing temperature to slow the molecular motion. Instead, can we utilize the thermal ellipsoids to visualize the molecular motion by analyzing the distances, angles, and torsions? If it works for the crystalline materials, it will be a real in-situ and real-time method to "see" solidstate molecular motion in atomic level. Furthermore, the motion amplitude or even direction can be easily tuned by the ambient temperature.

The best candidate to perform molecular motion should have a twisted structure which possesses large free volume. Tetraphenylethylene (TPE) is a typical molecular motor which shows aggregation-induced emission (AIE) effect. $\frac{13}{1}$ Previous works reported that the molecular motion in TPE showed a direct relationship with its photoluminescence (PL) properties. ${ }^{14}$ In solution, TPE has a vigorous motion and most of the excitedstate energy dissipates through nonradiative decay pathway. Then the formation of aggregates restricts the molecular motion and strong emission is triggered. By combining experimental and computational results, restriction of intramolecular motion (RIM) is proposed as the working mechanism of AIE. ${ }^{15,16}$ As shown in Fig. $1 A$, the molecular motion in TPE is divided into two categories, e.g. distance and angles. The change of distance can be caused by wagging and stretching, and the twisting/torsion/rocking are reflected in the variation of angles. Traditionally, the identification of these motions is based on speculation and they are seldom "seen" by a visual method. $\underline{17}$ In this work, the variable-temperature XRD was used to visualize the molecular motion in TPE crystal since the motion is sensitive to the surrounding temperature. As the results, with the temperature increasing from 150 to $298 \mathrm{~K}$, the ellipsoid becomes bigger which suggests a more intense atomic motion at $298 \mathrm{~K}$ (Fig. 1B). Meanwhile, the cell volume is enlarged to $955.66 \AA^{3}$ from $929.86 \AA^{3}$ accompanied with the intermolecular distance $(d)$ increasing from 7.62 to $7.84 \AA$. The interplanar angle $(\alpha)$ also exhibits a slight increase, suggesting the molecular motion involves both inter- and intramolecular modes (Fig. 1C). This work offers a simple and versatile technique to "see" the solidstate molecular motion which could be extensively applied in all crystalline molecules.
A suitable crystal of TPE was picked out by microscopy to carry out XRD under various temperature from $150 \mathrm{~K}\left(-123^{\circ} \mathrm{C}\right)$ to $298 \mathrm{~K}\left(25^{\circ} \mathrm{C}\right)$ with an interval of $15 \mathrm{~K}$. Its crystal structures at each temperature were all perfectly determined without any disorder (Fig. S1-11). Firstly, the double bond-related motions were analyzed and the changes of bond length and dihedral angle to temperature were plotted (Fig. 2). Fig. 2A suggested that the double-bond length (C13-C14) decreased from 1.357 to $1.350 \AA$ along with raising the temperature from $150 \mathrm{~K}$ to room temperature (RT). At the same time, torsion angle of double bond (C1C13C7 $\square \mathrm{C} 15 \mathrm{C} 14 \mathrm{C} 21)$ increased from 8.9 to $9.9^{\circ}$, resulting in a more twisted conformation (Fig. 2B). It is deduced that the vigorous motion at high temperature results in a twisted double bond. However, the twisted ethylene group will increase the intramolecular space and decrease the steric hindrance, then the atoms $\mathrm{C} 13$ and $\mathrm{C} 14$ get close to each other to maintain the electronic nature of double bond. That is the reason why the doublebond length becomes shorter and shorter along with the raising of temperature.

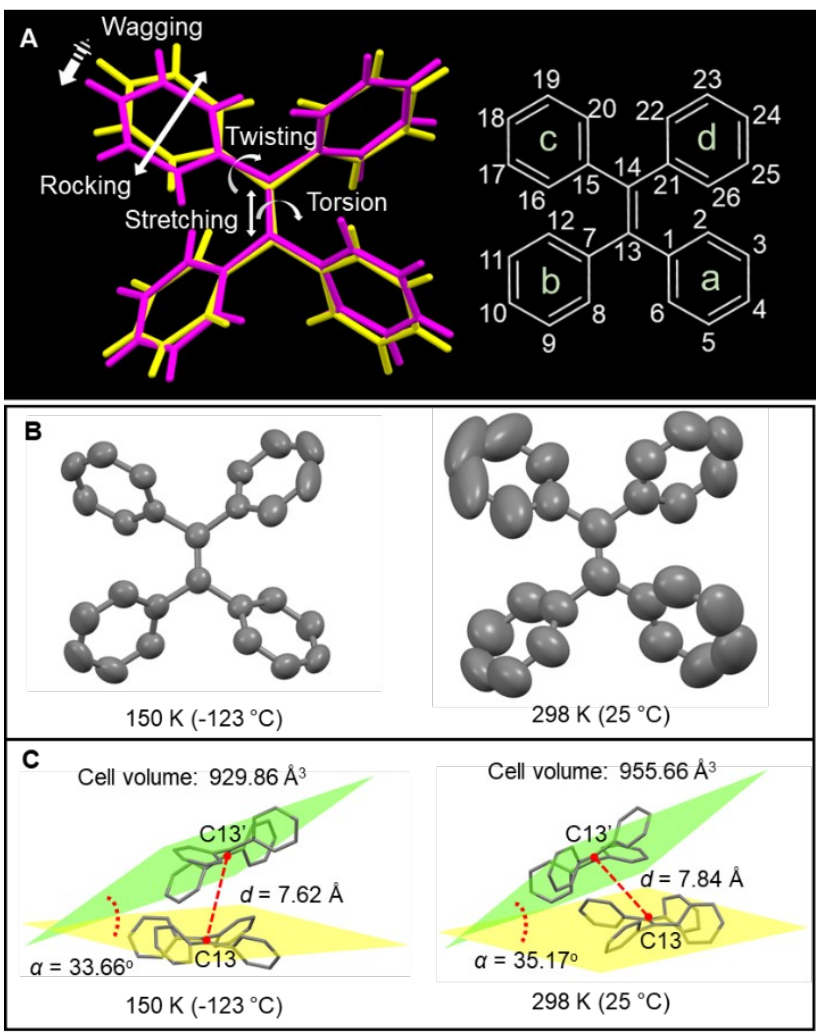

Figure. 1 (A) Models of molecular motions and atomic labels for TPE; (B) The thermal ellipsoid of TPE with $90 \%$ probability level $x$ at 150 and 298 K; (C) Cell volume, intermolecular distance and angle of TEP at $150 \mathrm{~K}$ and $298 \mathrm{~K}$. 

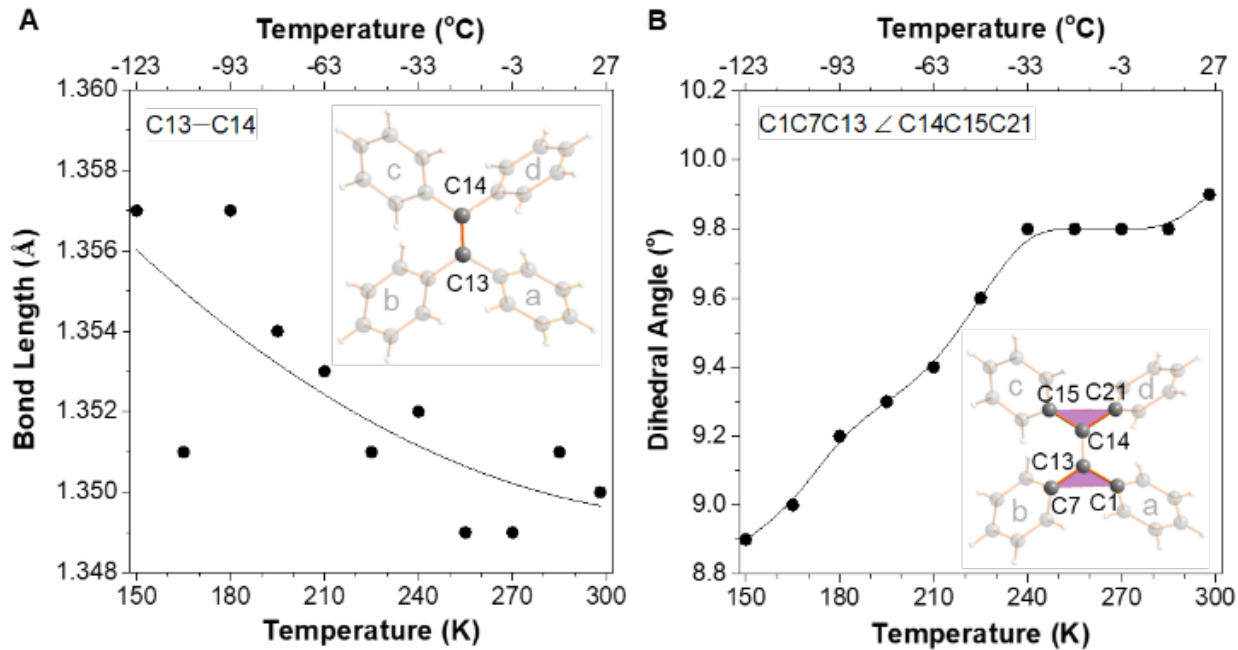

Figure. 2 Plots of the changes of $(A)$ double-bond $(\mathrm{C} 13=\mathrm{C} 14)$ length and $(\mathrm{B})$ double-bond torsion angle (planes $\mathrm{C} 1 \mathrm{C} 13 \mathrm{C} 7$ to $\mathrm{C} 15 \mathrm{C} 14 \mathrm{C} 21$ ) along with the temperature.

In addition to the double bond-related motions, the torsion angles of four peripheral phenyl rings $a$, $b, c$, and $d$ were also analyzed (Fig. 3). Rings $c$ and $d$ were connected by atom $\mathrm{C} 14$ which showed a large steric hindrance, that is the same situation in rings $b$ and $a$. With the temperature rising from 150 to $298 \mathrm{~K}$, the torsion angle of ring $c$ (C16C15C14C13) increased for $1.2^{\circ}$ accompanied with the decrease of that in ring $d$ $\left(\mathrm{C} 22 \mathrm{C} 21 \mathrm{C} 14 \mathrm{C} 13\right.$ ) (around $\left.-0.5^{\circ}\right)$. Similarly, there is a $3.0^{\circ}$ increase in torsion angle $\mathrm{C} 8 \mathrm{C} 7 \mathrm{C} 13 \mathrm{C} 14$ (ring b) and the torsion angle of ring a (C2C1C13C14) simultaneously decreased from $47.8^{\circ}$ to $45.4^{\circ}$. Obviously, the steric hindrance between rings $b$ and $c$ (also $a$ and $d$ ) is smaller than that of rings $c$ and $d$ ( $a$ and $b)$, that is the reason why the variation tendency of ring $c$ is similar to ring $b$ but opposite to ring $d$ so as to decrease the intramolecular steric hindrance. Meanwhile, rings a and $d$ turned to be planar concurrently and $b$ and $c$ became more twisted. Overall, the peripheral phenyl rings as a whole becomes more twisted with the increase of temperature $\left(\Delta=1.2^{\circ}-0.5^{\circ}+\right.$ $3.0^{\circ}-2.4^{\circ}=1.3^{\circ}$ ).
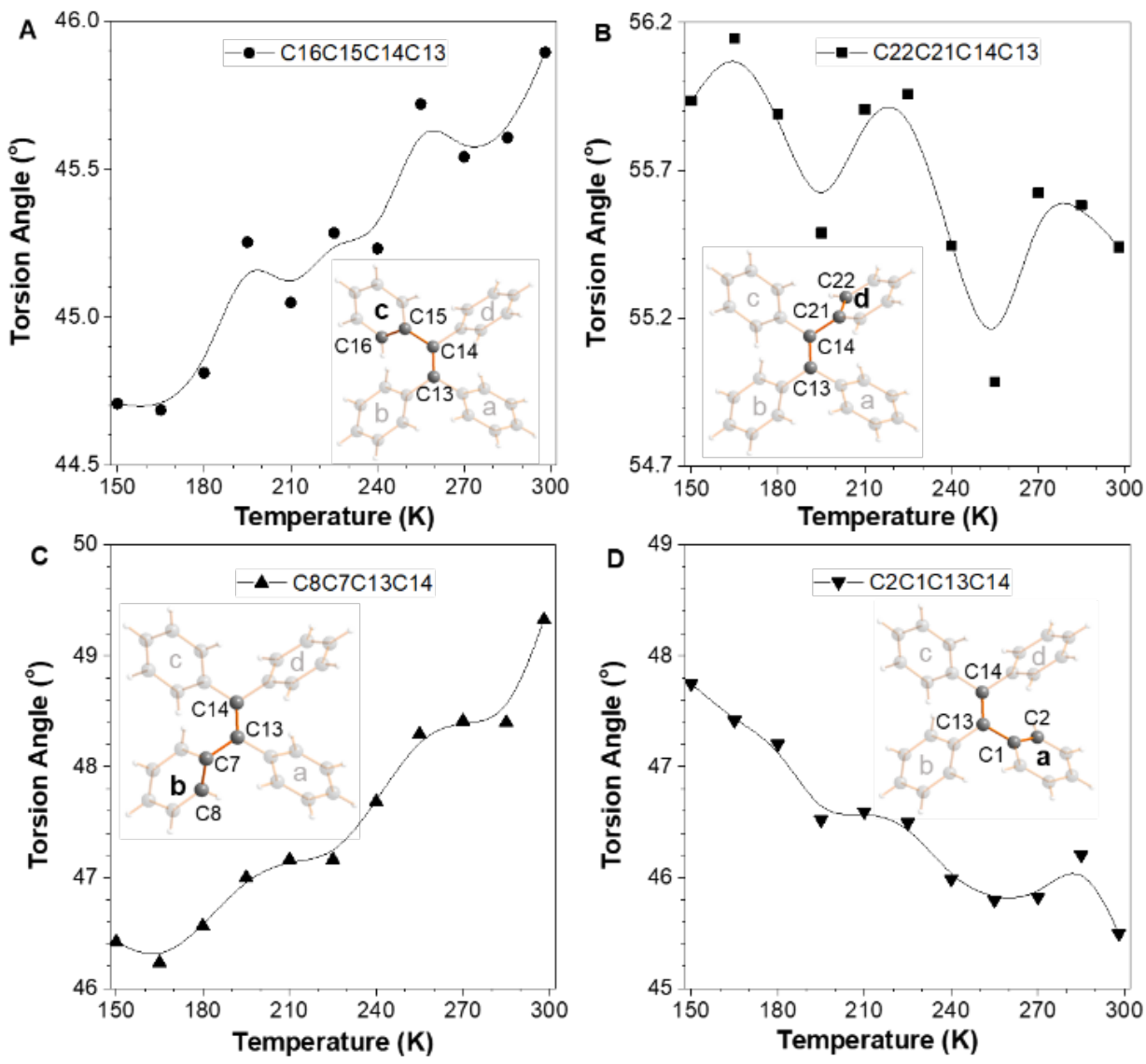
Figure. 3 The plots of torsion angle of (A) ring $c,(B)$ ring $d,(C)$ ring $b$, and (D) ring $a$ in TPE versus the change of temperature.

Above mentioned results showed the "static" molecular motion with the raising of temperature. In other words, the motion is represented by the conformational change under different temperature. How about the "dynamic" molecular motion at each temperature? In order to answer this question, the sites of the molecule at different temperature were all split and refined over two sites with site occupancy factors closed to 0.5 , which represented the two extreme positions for the motion. The distance between these two positions can reflect the amplitude of "dynamic" molecular motion. As shown in Fig. 4, the outermost hydrogens of $\mathrm{H} 18, \mathrm{H} 24, \mathrm{H} 10$, and $\mathrm{H} 4$ in peripheral phenyl rings were split to generate two extreme positions $A$ and $B$. The distances of $H 18 A$ vs $\mathrm{H} 18 \mathrm{~B}, \mathrm{H} 24 \mathrm{~A}$ vs $\mathrm{H} 24 \mathrm{~B}, \mathrm{H} 10 \mathrm{~A}$ vs $\mathrm{H} 10 \mathrm{~B}$ and $\mathrm{H} 4 \mathrm{~A}$ vs $\mathrm{H} 4 \mathrm{~B}$ are $0.67,0.44,0.44$, and $0.46 \AA$ at $150 \mathrm{~K}$, respectively. Then the rocking of the phenyl rings becomes more and more vigorous with the increase of testing temperature. At RT, the above mentioned distances increase to $0.89,0.55,0.59$, and $0.59 \AA$, respectively. For most of AlEgens, their solid-state photoluminescence is enhanced once their surrounding temperature is decreased which is usually ascribed to the effect of restriction of intramolecular motion, indicating the low temperature could partially freeze the molecular motions. Although the photoluminescence-related motions are in the excited state, the XRD results in this work provide compelling evidence for the RIM mechanism.

In summary, by means of XRD, the solid-state molecular motion in representative AIEgen of TPE was visualized successfully. Five kinds of motions, e.g. bond stretching, double-bond torsion, phenylring twisting, rocking, and wagging, have been monitored in this work. From the viewpoint of thermodynamics, these molecular motions were systematically divided into "static" and "dynamic" modes. In terms of the static motions, with the increase of ambient temperature, the middle double bond in TPE became short and twisted. Meanwhile, the torsion angles of peripheral phenyl rings also showed increase or decrease but all the phenyl rings as a whole was found to be more twisted. Dynamic molecular motion was also observed through crystal splitting and refining, which is represented by the rocking and wagging. As expected, these kinds of dynamic motions tended to be more and more vigorous with the raising of temperature, which provided direct evidence for the RIM mechanism of AIE. It is noteworthy that intermolecular motions were also observed in TPE crystals in addition to these intramolecular modes. In general, the complicated molecular motions were clearly visualized by a common structural determination technique, offering a new platform to "see" the microcosmic motions. 

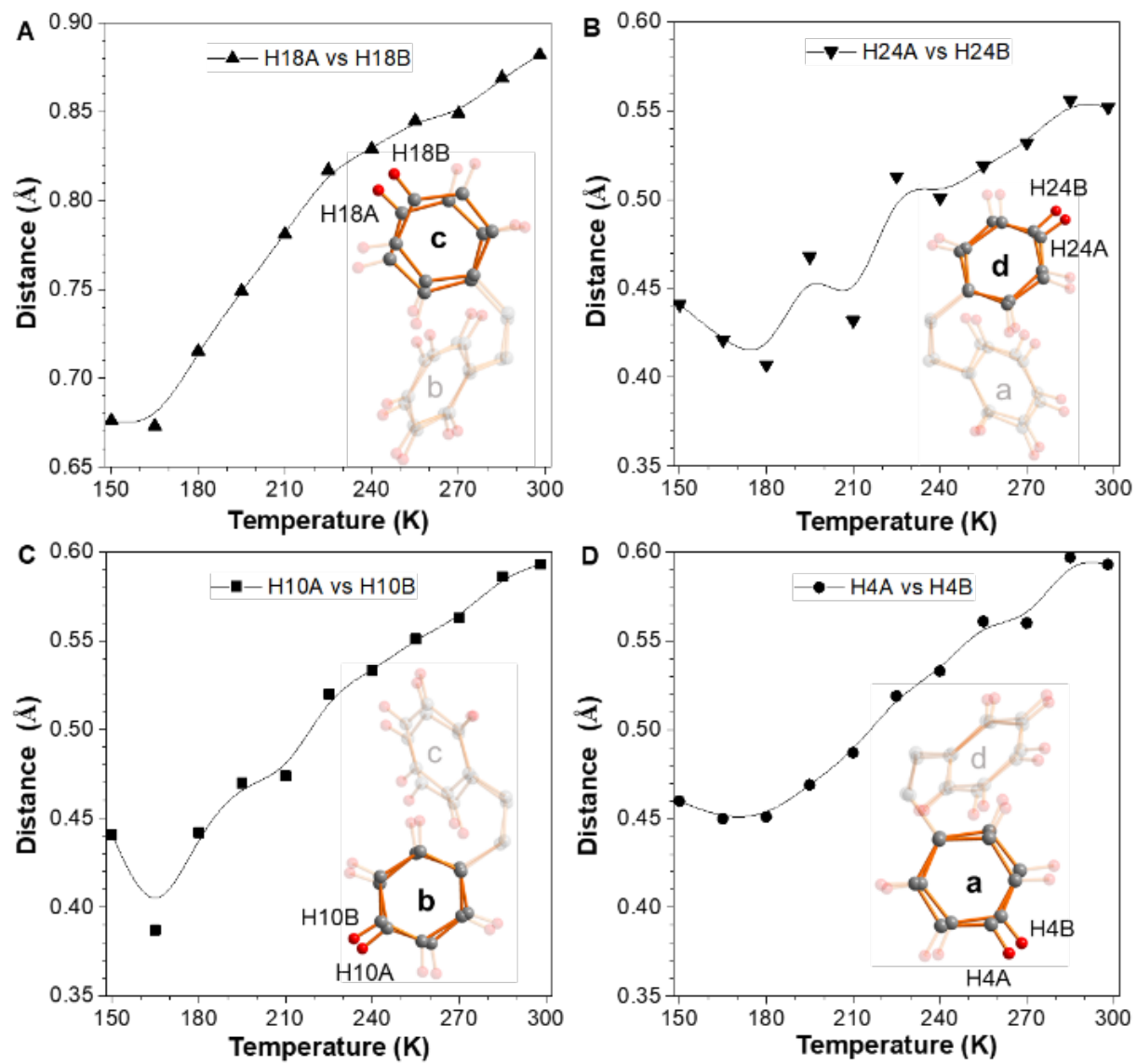

Figure. 4 The vibration amplitude of the terminal hydrogens (A) H18, (B) H24, (C) H1O, and (D) H4 under different temperature.

\section{ASSOCIATED CONTENT}

\section{Supporting Information}

The asymmetric unit of TPE at different temperature can be found in the supplementary information. The Supporting Information is available free of charge on the ACS Publications website.

\section{AUTHOR INFORMATION}

\section{Corresponding Author}

tangbenz@ust.hk (B.Z. Tang)

\section{Author Contributions}

\# J. Zhang, H. Zhang, and J. Liu contributed equally to this work.

\section{Notes}

The authors declare no competing financial interests.

\section{ACKNOWLEDGMENT}

We are grateful for financial support from the National Science Foundation of China (21788102, 21671003), the Research Grants Council of Hong Kong (16308016, C6009-17G and A-HKUST 605/16), the Innovation and Technology Commission (ITC-CNERC14SC01) and the Science

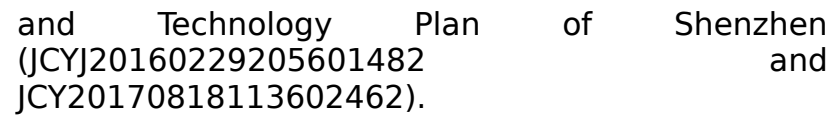

\section{REFERENCES}

(1) Gao, M.; Lu, C.; Jean-Ruel, H.; Liu, L. C.; Marx, A.; Onda, K.; Koshihara, S.; Nakano, Y.; Shao, X. F.; Hiramatsu, T.; Saito, G.; Yamochi, H.; Cooney, R. R.; Moriena, G.; Sciaini, G.; Miller, R. J. D. Nature 2013, 496, 343.

(2) Garcia-Lopez, V.; Chen, F.; Nilewski, L. G.; Duret, G.; Aliyan, A.; Kolomeisky, A. B.; Robinson, J. T.; Wang, G.; Pal, R.; Tour, J. M. Nature 2017, 548, 567.

(3) Liu, S.; Li, Y.; Zhang, H.; Zhao, Z.; Lu, X.; Lam, J. W. Y.; Tang, B. Z. ACS Mat. Lett. 2019, 425.

(4) Alam, P.; Leung, N. L. C.; Cheng, Y. H.; Zhang, H. K.; Liu, J. K.; Wu, W. J.; Kwok, R. T. K.; Lam, J. W. Y.; Sung, H. H. Y.; Williams, I. D.; Tang, B. Z. Angew. Chem. Int. Ed. 2019, 58, 4536.

(5) Comotti, A.; Bracco, S.; Sozzani, P. Acc. Chem. Re.s 2016, 49, 1701.

(6) Ryabchun, A.; Li, Q.; Lancia, F.; Aprahamian, I.; Katsonis, N. J. Am. Chem. Soc. 2019, 141, 1196.

(7) Liu, S. J.; Zhou, X.; Zhang, H. K.; Ou, H. L.; Lam, J. W. Y.; Liu, Y.; Shi, L. Q.; Ding, D.; Tang, B. Z. J. Am. Chem. Soc. 2019, 141, 5359.

(8) Kaleta, J.; Chen, J. W.; Bastien, G.; Dracinsky, M.; Masat, M.; Rogers, C. T.; Feringa, B. L.; Michl, J. J. Am. Chem. Soc. 2017, 139, 10486. 
(9) Pejov, L.; Panda, M. K.; Moriwaki, T.; Naumov, P. J. Am. Chem. Soc. 2017, 139, 2318.

(10) Zhang, H.; Sun, J. Z.; Liu, J.; Kwok, R. T. K.; Lam, J. W. Y.; Tang, B. Z. J. Appl. Phys. 2019, 126, 050901.

(11) Kudernac, T.; Ruangsupapichat, N.; Parschau, M.; Macia, B.; Katsonis, N.; Harutyunyan, S. R.; Ernst, K. H.; Feringa, B. L. Nature 2011, 479, 208.

(12) Shustova, N. B.; Ong, T. C.; Cozzolino, A. F.; Michaelis, V. K.; Griffin, R. G.; Dinca, M. J. Am. Chem. Soc. 2012, 134, 15061.

(13) Mei, J.; Leung, N. L. C.; Kwok, R. T. K.; Lam, J. W. Y.; Tang, B. Z. Chem. Rev. 2015, 115, 11718.

(14) Chen, J. W.; Law, C. C. W.; Lam, J. W. Y.; Dong, Y. P.; Lo, S. M. F.; Williams, I. D.; Zhu, D. B.; Tang, B. Z. Chem. Mater. 2003, 15, 1535.

(15) Leung, N. L. C.; Xie, N.; Yuan, W. Z.; Liu, Y.; Wu, Q. Y.; Peng, Q.; Miao, Q.; Lam, J. W. Y.; Tang, B. Z. Chem.: Eur. J. 2014, 20, 15349.

(16) Zhang, H.; Liu, J.; Du, L.; Ma, C.; Leung, N. L. C.; Niu, Y.; Qin, A.; Sun, J.; Peng, Q.; Sung, H. H. Y.; Williams, I. D.; Kwok, R. T. K.; Lam, J. W. Y.; Wong, K. S.; Phillips, D. L.; Tang, B. Z. Mater. Chem. Front. 2019, 3, 1143.

(17) Gao, Y. J.; Chang, X. P.; Liu, X. Y.; Li, Q. S.; Cui, G. L.; Thiel, W. J. Phys. Chem. A 2017, 121, 2572. 